INRA Prod. Anim.,

2012,25 (4), 351-360

\title{
Analyse quantitative de l'effet des bactéries probiotiques sur les fermentations dans le rumen et les performances des bovins en production
}

\author{
A. LETTAT $1,2,3$, C. MARTIN 1,2, C. BERGER 4 , P. NOZIÈRE 1,2 \\ ${ }^{1}$ INRA, UMR1213 Herbivores, F-63122 Saint-Genès-Champanelle, France \\ ${ }^{2}$ Clermont Université, VetAgro Sup, UMR Herbivores, BP 10448, F-63000 Clermont-Ferrand, France \\ ${ }^{3}$ Danisco France SAS, Zone d'Activités de Buxières, BP 10, F-86220 Dangé Saint Romain, France \\ ${ }^{4}$ Danisco France SAS, 20 rue Brunel, F-75017 Paris, France \\ Courriel : pierre.noziere@clermont.inra.fr
}

L'acidose ruminale latente est fortement présente dans les élevages intensifs de ruminants et engendre des pertes économiques importantes. Pour la prévenir, l'utilisation en tant qu'additifs alimentaires de bactéries probiotiques, seules ou associées à la levure $S$. cerevisiae, fait l'objet de nombreuses recherches. Il convient d'en évaluer les effets sur les fermentations dans le rumen et sur les performances de production des bovins.

L'intensification des conduites alimentaires des bovins laitiers et à viande est à l'origine de l'acidose ruminale latente. Cette pathologie d'origine digestive a déjà suscité une intense activité de recherche comme en témoignent de nombreuses synthèses bibliographiques dont le dossier de la revue INRA Productions Animales publié en 2006 (Martin et al 2006, Peyraud et Apper-Bossard 2006, Sauvant et al 2006). Du fait de sa forte prévalence, estimée environ à $19 \%$ chez la vache laitière (Kleen et al 2009), et de ses conséquences négatives sur la santé et les performances animales (Khafipour et al 2009 a et b, Krause et al 2009), différentes stratégies ont été développées afin de prévenir l'acidose ruminale latente. Le premier levier d'action réside dans une formulation de la ration adaptée aux besoins de l'animal et un mode de distribution permettant une adaptation progressive de l'écosystème ruminal (Sauvant et Peyraud 2010). Lorsque le risque d'acidose demeure élevé malgré ces précautions, l'addition de substances tampons est recommandée pour stabiliser le $\mathrm{pH}$ ruminal et améliorer les performances animales (Meschy et al 2004). Enfin, l'utilisation d'additifs microbiens semble également constituer une solution efficace pour limiter les risques d'acidose latente chez le ruminant. Si les levures ont été largement étudiées (Chaucheyras-Durand et al 2008,
Desnoyers et al 2009, ChaucheyrasDurand et Durand 2010), la supplémentation en Bactéries Probiotiques (BP) constitue un nouveau champ de recherche particulièrement intéressant car contrairement aux levures qui ne survivent pas longtemps dans le rumen, les bactéries, plus particulièrement celles d'origine ruminale, pourraient mieux s'adapter aux conditions anaérobies du rumen et produire de meilleurs effets lorsque l'animal est en situation d'acidose.

L'utilisation des BP chez le ruminant adulte a principalement été proposée dans le but d'améliorer les performances zootechniques du bovin laitier (Stein et al 2006, Raeth-Knight et al 2007 , Lehloenya et al 2008b) ou à viande (Lehloenya et al 2008a, Vasconcelos et al 2008) et de réduire le portage de bactéries pathogènes (E. coli O157:H7, Salmonelles) chez les bovins à l'engrais (Peterson et al 2007, Stephens et al 2007a, Stephens et al 2007b). Les BP utilisées seules ou en association sont principalement des bactéries lactiques et propioniques respectivement issues des genres Lactobacillus et Propionibacterium. Théoriquement, l'utilisation conjointe de ces deux types bactériens permettrait, dans le rumen, une production accrue de lactate par les lactobacilles qui serait ensuite métabolisé en propionate par les propionibactéries. Le propionate étant le principal précurseur du glucose via la néoglucogenèse (Brockman 1990, Loncke et al 2009), cela pourrait in fine contribuer à améliorer la production laitière (Francisco et al 2002, Stein et al 2006, Weiss et al 2008) et la synthèse de protéines musculaires chez le bovin à viande (Lehloenya et al 2008a). Par ailleurs, la production de lactate par les lactobacilles permettrait de stimuler la croissance des bactéries utilisatrices de lactate. Ce mécanisme s'apparente à un effet vaccin permettant à la flore utilisatrice de lactate d'être efficace et donc de prévenir l'accumulation d'acide lactique lors de situations nutritionnelles conduisant à l'apparition de l'acidose (Nocek et al 2002, Nocek et al 2003, Meissner et al 2010). L'objectif de cette synthèse est donc de vérifier dans quelle mesure ces bénéfices théoriques sont observés en pratique, en particulier dans des situations d'acidose latente.

Cet article fournit une revue quantitative de l'intérêt d'une supplémentation en BP, seules ou associées à la levure Saccharomyces cerevisiae (SC) dans l'alimentation des ruminants en situation d'acidose latente. La première partie est consacrée aux effets des BP sur les performances zootechniques des animaux en dissociant les bovins lai- 
Tableau 1. Caractéristiques des expériences menées sur vaches laitières et incluses dans la méta-analyse.

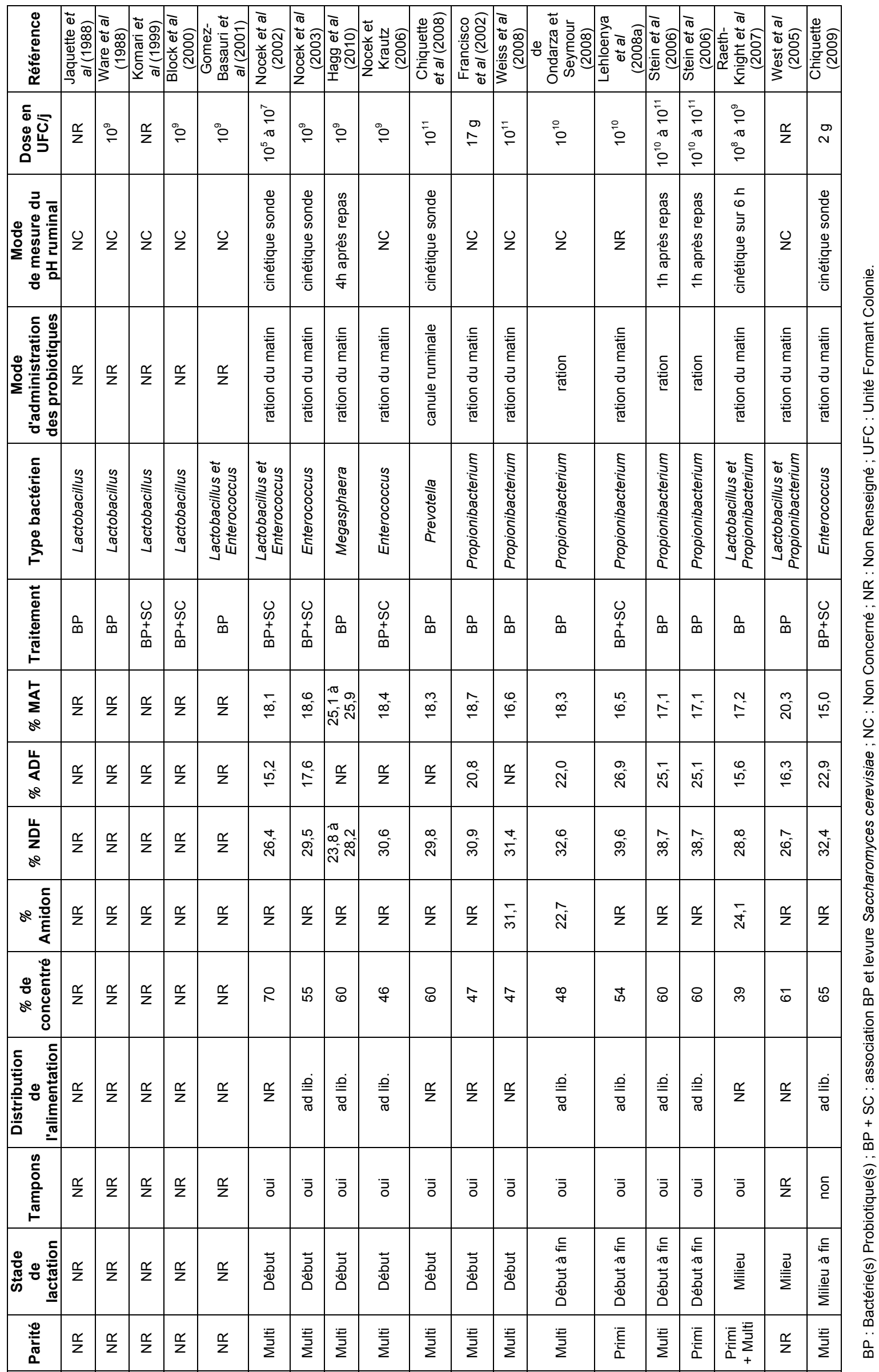


tiers et à viande. L'effet des BP sur les paramètres du rumen $(\mathrm{pH}$, profils fermentaires et microbiens) est abordé dans une deuxième partie.

\section{1 / Les données rassemblées et les méthodes d'analyse}

Nous avons rassemblé tous les essais in vivo dans lesquels les effets d'un apport de BP sur les performances zootechniques et/ou les paramètres fermentaires du rumen ont été étudiés avec des rations de base présentant un risque acidogène potentiel $(\geq 40 \%$ de concentré dans la ration; Sauvant et Peyraud 2010). Devant le manque de données relatives à l'utilisation des BP seules, nous avons également pris en compte les essais dans lesquels les BP ont été associées à la levure $S$. cerevisiae. L'ensemble des résultats de ces essais a été organisé dans une base de données regroupant un total de 33 expériences correspondant à 82 traitements. Les expériences incluses dans la méta-analyse sont décrites dans les tableaux 1 et 2 .

Chez la vache laitière, 19 études ont été recensées dont 5 publiées seulement sous forme de résumés (régime, $\%$ de concentré, dose de probiotiques non renseignés). Les rations offertes aux animaux comportaient en moyenne $56 \pm 9.6 \%$ de concentré (de 39 à $70 \%$ ). Les proportions de NDF, ADF et MAT étaient respectivement de $31,2 \pm 4,7 \%$; $20,7 \pm 4,3 \%$; et $17,7 \pm 3 \%$. La plupart des rations comprenait entre 0,5 et $1,85 \%$ de tampons (carbonate de calcium pour la majorité).

Pour les bovins à viande, nous avons recensé 14 expériences (37 traitements), dans lesquelles les animaux étaient alimentés avec des rations comportant en moyenne $87 \pm 12,8 \%$ de concentré (de 56 à 100\%). Les proportions de NDF, ADF et MAT n'étaient décrites que dans 6 expériences ( 4 pour le NDF), tandis que celle d'amidon n'est pas disponible. L'essentiel des rations comprenait des tampons chimiques et/ou des antibiotiques promoteurs de croissance.

Sur les 33 expériences rassemblées, seules 15 rapportaient l'effet des BP seules ou en association avec $S$. cerevisiae sur le $\mathrm{pH}$ et/ou les fermentations ruminales chez la vache laitière ou le bovin en croissance. Les rations comportaient une proportion moyenne en concentré de $69 \pm 17 \%$ (de 39 à 100\%). Les proportions de NDF, ADF et MAT étaient respectivement de $29 \pm 6,5 \%$; $17,5 \pm 4,3 \%$ et $18,1 \pm 4,1 \%$. Parmi ces études, 11 avaient pour but d'évaluer l'efficacité des $\mathrm{BP}$ à maintenir le $\mathrm{pH}$ ruminal.
Nous avons quantifié les réponses zootechniques et digestives des animaux à l'apport de BP seules ou à l'apport simultané de BP associées à $S$. cerevisiae $(\mathrm{BP}+\mathrm{SC})$. Les analyses ont été réalisées par analyse de variance en testant comme effets fixes, l'expérience, l'espèce animale (hiérarchisée dans l'effet expérience), la supplémentation en BP $(0$ vs 1$)$ et la nature de la supplémentation (BP vs $\mathrm{BP}+\mathrm{SC}$, hiérarchisée dans l'effet supplémentation). Les réponses zootechniques analysées ont été, pour la vache laitière, la Production Laitière (PL), la Matière Sèche Ingérée (MSI), les Taux Butyreux (TB), Protéique (TP) et de Lactose (TL) ainsi que le nombre de cellules somatiques du lait; pour les bovins à l'engrais ou en croissance, la MSI, le Gain Moyen Quotidien (GMQ) et l'efficacité alimentaire (GMQ/MSI). Les réponses digestives analysées ont été le pH, les concentrations ruminales en AGV totaux, lactate et ammoniaque et les proportions molaires des AGV.

\section{2 / Performances zootech- niques}

\section{1 / Vaches laitières}

Peu d'études ont été entreprises pour évaluer l'effet des BP sur les performances zootechniques des vaches laitières même s'il est bien établi que l'acido- se latente a des conséquences négatives sur les performances animales : ingestion, production et composition du lait (Nocek 1997, Kleen et al 2003, Gozho et al 2005, 2006, 2007).

Comparée aux vaches témoins (tableau 3), l'utilisation des BP seules (qu'elles soient lactiques, propioniques ou autres) n'a en moyenne pas modifié de façon significative l'ingestion $(-0,27 \mathrm{~kg} / \mathrm{j})$, la PL $(+0,89 \mathrm{~kg} / \mathrm{j})$, ni la composition du lait à l'exception du nombre de cellules somatiques qui tend à diminuer. Les effets positifs les plus nets sur les performances zootechniques des animaux (MSI et PL) ont été observés avec les bactéries lactiques seules (lactobacilles et entérocoques). Toutefois, dans l'étude de Gomez-Basauri et al (2001) l'effet observé pourrait être non spécifique des BP du fait de la supplémentation de la ration en mannanoligosaccharide (polymère de mannose extrait de la paroi de $S$. cerevisiae). Concernant les bactéries propioniques, à l'exception d'un essai non publié dans une revue scientifique (West et al 2005), l'utilisation de Propionibacterium freudenreichii PF24 ou P169, seules ou en association avec des bactéries lactiques, n'a amélioré ni l'ingestion, ni la production laitière (Francisco et al 2002, Raeth-Knight et al 2007, Weiss et al 2008). De la même manière, aucun effet $n$ 'a été observé sur les performances zootechniques des vaches suite à la supplémentation des rations avec Prevotella bryantii 25A

Figure 1. Relation entre Production Laitière $(P L)$ et Matière Sèche $(M S)$ ingérée chez des vaches laitières non supplémentées (O) ou supplémentées en bactéries probiotiques seules (๑) ou associées à la levure S. cerevisiae (๑). Les traitements d'une même étude sont reliés entre eux.

Liste des publications incluses dans la méta-analyse : Ware et al 1988, Block et al 2000, Gomez-Basauri et al 2001, Francisco et al 2002, Nocek et al 2003, West et al 2005, Nocek et Kautz 2006, Raeth-Knight et al 2007, Chiquette et al 2008, Weiss et al 2008, Hagg et al 2010, Lettat 2011.

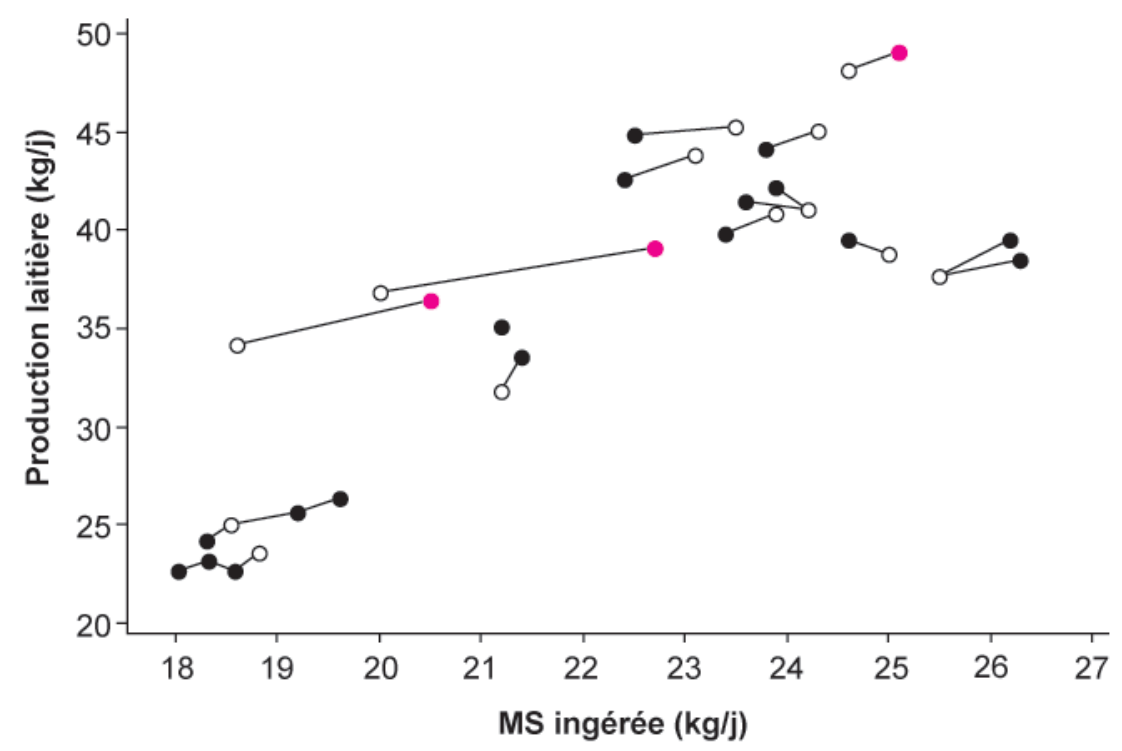


Tableau 2. Caractéristiques des expériences menées sur bovins à viande et incluses dans la méta-analyse.

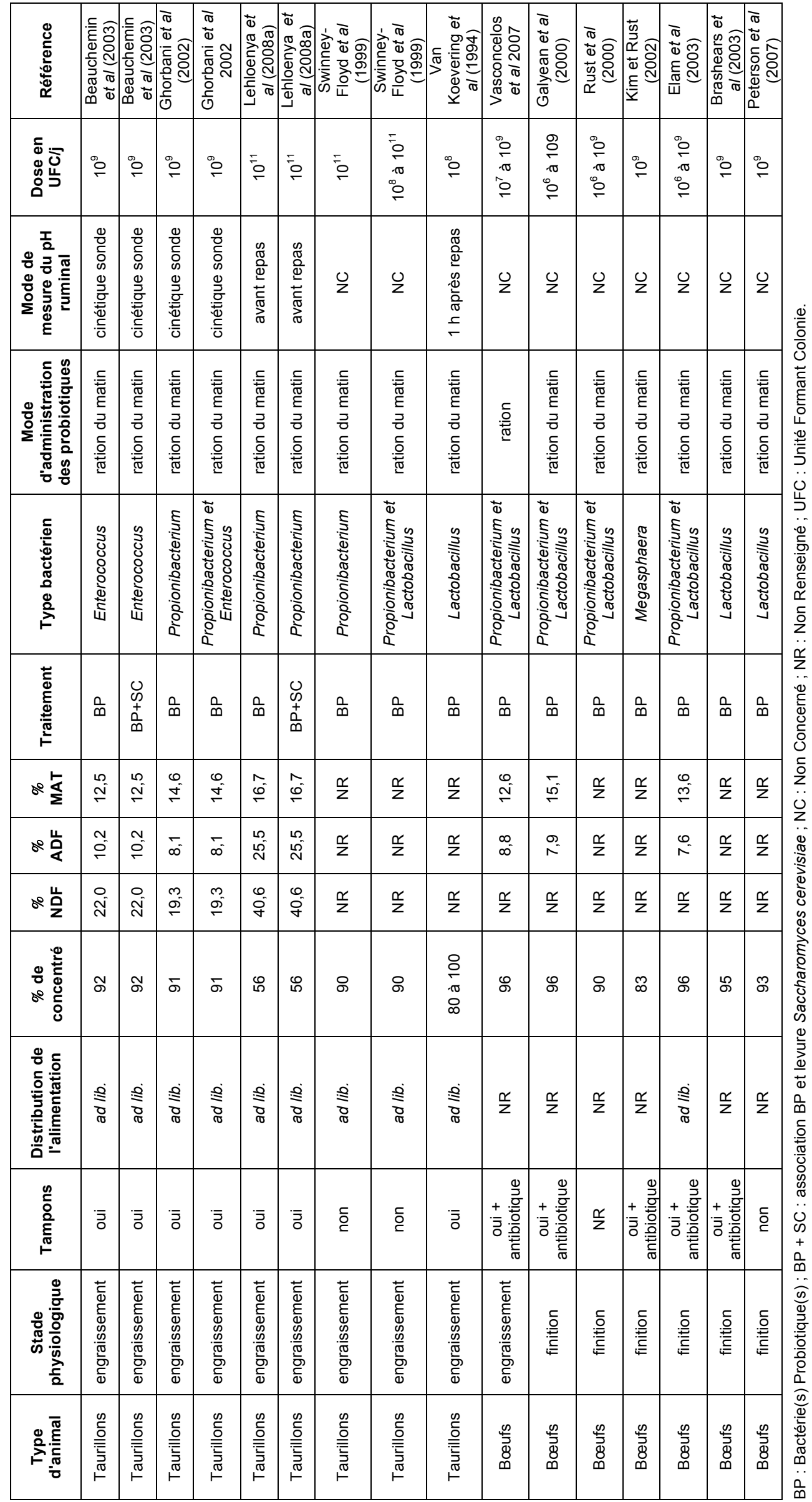


(Chiquette et al 2008) ou Megasphaera elsdenii NCIMB 41125 (Hagg et al 2010).

En revanche, l'utilisation de BP (lactiques ou propioniques) conjointement avec la levure $S$. cerevisiae a permis d'augmenter la MSI $(+1,70 \mathrm{~kg} / \mathrm{j})$, la PL $(+2,36 \mathrm{~kg} / \mathrm{j})$, et le TP $(+1 \mathrm{~g} / \mathrm{kg})$ (tableau 3). Puisque l'association BP + $\mathrm{SC}$ produit des effets supérieurs à ceux observés pour les bactéries seules, un effet spécifique de $S$. cerevisiae est probable (Desnoyers et al 2009, Chaucheyras-Durand et Durand 2010) sans pour autant exclure une synergie entre levures et bactéries. Cependant, aucun essai n'a été directement focalisé sur l'étude de cette interaction entre les deux types d'additifs microbiens. Enfin, quel que soit le type de supplémentation en probiotiques (BP ou BP $+\mathrm{SC}$ ), toutes les variations de PL observées ont été liées à des variations de l'ingestion, la réponse intra-essai étant de $+1,0 \pm$ $0,2 \mathrm{~kg}$ lait/kg MS ingérée (figure 1).

\section{2 / Bovins en croissance et à l'engrais}

En moyenne, aucun effet des BP seules ou en association n'a été observé sur l'ingestion et l'efficacité alimentaire (tableau 4). L'utilisation des bactéries lactiques seules ou associées à des propionibactéries constitue l'essentiel des données rassemblées. Les effets positifs les plus notables sur les performances (GMQ, efficacité) des bovins en croissance ont été observés avec l'association de bactéries lactiques et propioniques (Swinney-Floyd et al 1999). La même tendance est observée sur les bovins en fin d'engraissement (Rust et al 2000). Les rares données sur le rumen disponibles au sein de ces essais ne permettent pas de dégager les mécanismes d'action impliqués.

En conclusion, chez la vache laitière, l'amélioration de la production laitière n'est avérée que par l'utilisation des BP (bactéries lactiques ou propioniques) combinées à la levure $S$. cerevisiae. Ces effets sont liés à une augmentation de l'ingestion. Chez le bovin à viande, les effets positifs sur la croissance ont été essentiellement obtenus par l'apport de bactéries probiotiques associant des bactéries lactiques et des bactéries propioniques (Lactobacillus acidophilus et $P$. freudenreichii). L'intensité des effets des BP apportées seules ou en associa- tion avec SC sur les performances des bovins pourrait aussi s'expliquer par la présence quasi systématique de tampons et/ou de promoteurs de croissance dans les rations.

\section{3 / Fermentations ruminales}

\section{1 / Effets sur le pH ruminal}

La méta-analyse des effets des BP sur les fermentations ruminales a été faite sur l'ensemble des données provenant des bovins laitiers et à viande (tableau 5). Chez la vache laitière, aucun effet significatif sur le $\mathrm{pH}$ ruminal n'a été observé en utilisant des bactéries propioniques, que ce soit seules (Stein et al 2006) ou en association avec des bactéries lactiques (Raeth-Knight et al 2007) ; de même, aucun effet sur le $\mathrm{pH}$ n'a été observé suite a une supplémentation avec $P$. bryantii et $M$. elsdenii (Chiquette et al 2008, Hagg et al 2010). Les seules études rapportant un effet significatif sur le $\mathrm{pH}$ ont été obtenues par l'association de bactéries lactiques avec la levure $S$. cerevisiae (Nocek et al 2003, Chiquette 2009). Chez les bovins en croissance, aucun effet significatif

Tableau 3. Effet des bactéries probiotiques seules ou associées à la levure S. cerevisiae sur les performances zootechniques des vaches laitières.

\begin{tabular}{|c|c|c|c|c|c|c|c|c|}
\hline & & \multirow[b]{2}{*}{ Témoin } & \multirow[b]{2}{*}{ Traité } & \multirow[b]{2}{*}{ Variation } & \multirow[b]{2}{*}{$N_{\exp }$} & \multirow[b]{2}{*}{ ETR } & \multicolumn{2}{|c|}{ Effets statistiques } \\
\hline & & & & & & & $\begin{array}{c}\text { Probiotique } \\
\text { (Traité vs Témoin) }\end{array}$ & $\begin{array}{c}\text { Nature du } \\
\text { probiotique } \\
\text { (Variation BP vs } \\
\text { Variation BP + SC) }\end{array}$ \\
\hline \multirow{2}{*}{ MSI (kg/j) } & BP & 23,8 & 23,6 & $-0,27$ & 11 & \multirow{2}{*}{0,50} & \multirow{2}{*}{$P=0,006$} & \multirow{2}{*}{$P=0,001$} \\
\hline & $\mathrm{BP}+\mathrm{SC}$ & 21,1 & 22,8 & 1,70 & 3 & & & \\
\hline \multirow{2}{*}{ PL (kg/j) } & BP & 37,5 & 38,4 & 0,89 & 17 & \multirow{2}{*}{1,00} & \multirow{2}{*}{$P<0,001$} & \multirow{2}{*}{$P=0,05$} \\
\hline & $B P+S C$ & 33,3 & 35,7 & 2,36 & 5 & & & \\
\hline \multirow{2}{*}{ TB (\%) } & BP & 3,56 & 3,52 & $-0,04$ & 17 & \multirow{2}{*}{0,18} & \multirow{2}{*}{ NS } & \multirow{2}{*}{ NS } \\
\hline & $\mathrm{BP}+\mathrm{SC}$ & 4,01 & 3,97 & $-0,04$ & 4 & & & \\
\hline \multirow{2}{*}{ TP (\%) } & BP & 2,99 & 3,01 & 0,02 & 13 & \multirow{2}{*}{0,05} & \multirow{2}{*}{$P=0,01$} & \multirow{2}{*}{$P=0,06$} \\
\hline & $\mathrm{BP}+\mathrm{SC}$ & 3,08 & 3,18 & 0,10 & 5 & & & \\
\hline \multirow{2}{*}{ TL (\%) } & BP & 4,81 & 4,86 & 0,05 & 9 & \multirow{2}{*}{0,09} & \multirow{2}{*}{ NS } & \multirow{2}{*}{ NS } \\
\hline & $B P+S C$ & 4,61 & 4,72 & 0,11 & 2 & & & \\
\hline \multirow{2}{*}{$\begin{array}{l}\text { Cellules } \\
\times 10^{3} / \mathrm{mL}\end{array}$} & $B P$ & 164 & 95 & -69 & 7 & \multirow{2}{*}{56} & \multirow{2}{*}{ NS } & \multirow{2}{*}{$P=0,09$} \\
\hline & $B P+S C$ & 181 & 238 & 57 & 2 & & & \\
\hline
\end{tabular}

$\mathrm{BP}=$ Bactérie(s) Probiotique(s) seule(s) $; \mathrm{BP}+\mathrm{SC}=$ association BP et levure Saccharomyces cerevisiae $; \mathrm{PL}=$ Production Laitière $; \mathrm{MSI}=$ Matière Sèche Ingérée $; \mathrm{TB}=$ Taux Butyreux $; \mathrm{TP}=$ Taux Protéique $; \mathrm{TL}=\mathrm{Taux}$ de Lactose ; Cellules $=$ nombre de cellules somatiques; $N_{\exp }=$ nombre d'expériences incluses dans le dispositif ; ETR = Ecart Type Résiduel ; NS = Non Significatif. Les variations significatives par rapport au témoin sont en gras.

Liste des publications incluses dans la méta-analyse : Jaquette et al 1988, Ware et al 1988, Komari et al 1999, Block et al 2000, Gomez-Basauri et al 2001, Francisco et al 2002, Nocek et al 2003, West et al 2005, Nocek et Kautz 2006, Stein et al 2006, Raeth-Knight et al 2007, Chiquette et al 2008, de Ondarza et Seymour 2008, Lehloenya et al 2008b, Weiss et al 2008, Hagg et al 2010. 
Tableau 4. Effet des bactéries probiotiques seules ou associées à la levure S. cerevisiae sur l'ingestion, la croissance et l'efficacité alimentaire des bovins en croissance et à l'engrais.

\begin{tabular}{|c|c|c|c|c|c|c|c|c|}
\hline & \multirow[b]{2}{*}{ Témoin } & \multirow[b]{2}{*}{ Traité } & \multirow[b]{2}{*}{ Variation } & \multirow[b]{2}{*}{$N_{\exp }$} & \multirow[b]{2}{*}{ ETR } & \multicolumn{2}{|c|}{ Effets statistiques } \\
\hline & & & & & & & $\begin{array}{l}\text { Probiotique } \\
\text { (Traité vs } \\
\text { Témoin) } \\
\end{array}$ & $\begin{array}{c}\text { Nature du probiotique } \\
\text { (Variation BP vs } \\
\text { Variation BP + SC) } \\
\end{array}$ \\
\hline \multirow{2}{*}{ MSI (kg/j) } & $\mathrm{BP}$ & 9,28 & 9,34 & 0,06 & 19 & \multirow{2}{*}{0,28} & \multirow{2}{*}{ NS } & \multirow{2}{*}{ NS } \\
\hline & $\mathrm{BP}+\mathrm{SC}$ & 7,95 & 7,75 & $-0,20$ & 1 & & & \\
\hline \multirow{2}{*}{$\mathrm{GMQ}(\mathrm{kg} / \mathrm{j})$} & $\mathrm{BP}$ & 1,42 & 1,51 & 0,09 & 19 & \multirow{2}{*}{0,08} & \multirow{2}{*}{$P=0,02$} & \multirow{2}{*}{ NS } \\
\hline & $B P+S C$ & 0,67 & 0,87 & 0,20 & 1 & & & \\
\hline \multirow{2}{*}{ Efficacité } & $\mathrm{BP}$ & 0,16 & 0,18 & 0,01 & 19 & \multirow{2}{*}{0,02} & \multirow{2}{*}{ NS } & \multirow{2}{*}{ NS } \\
\hline & $B P+S C$ & 0,08 & 0,11 & 0,03 & 1 & & & \\
\hline
\end{tabular}

$\mathrm{BP}=$ Bactérie(s) Probiotique(s) seule(s) ; BP + SC = association BP et levure Saccharomyces cerevisiae ; MSI = Matière Sèche Ingérée ; $G M Q=$ Gain Moyen Quotidien ; $N_{\exp }=$ nombre d'expériences incluses dans le dispositif ; ETR = Ecart Type Résiduel ; NS = Non Significatif.

Liste des publications incluses dans la méta-analyse: Van Koevering et al 1994, Swinney-Floyd et al 1999, Galyean et al 2000, Kim et al 2000, Rust et al 2000, Beauchemin et al 2003, Brashears et al 2003, Elam et al 2003, Peterson et al 2007, Vasconcelos et al 2007.

des BP n'a été observé sur le $\mathrm{pH}$ ruminal, quelle que soit la composition des BP étudiées : bactérie propionique seule, P. acidopropionici P15 (Ghorbani et al 2002) ou P. freudenreichii P169 (Lehloenya et al 2008a), bactérie propionique associée à la bactérie lactique E. faecium 212 (Ghorbani et al 2002) ou à la levure $S$. cerevisiae (Lehloenya et al 2008a), bactérie lactique seule (EF212) ou associée à la levure $S$. cerevisiae (Beauchemin et al 2003).

En raison de la diversité des situations expérimentales selon les essais (types d'animaux, de régime, nature et dose des BP utilisées...), il est difficile de tirer une conclusion quant à l'efficacité relative des différents probiotiques pour limiter l'acidité dans le rumen. D'après les résultats rassemblés à la figure 2, nous émettons l'hypothèse selon laquelle la régulation du $\mathrm{pH}$ ruminal par les BP ne serait effective que lorsque le $\mathrm{pH}$ ruminal moyen avec la ration de base est suffisamment faible. Ainsi, dans la seule étude publiée ayant démontré un effet notable de la supplémentation en $\mathrm{BP}$ sur le $\mathrm{pH}$ (Chiquette 2009), aucun antibiotique ni tampon n'était inclus dans la ration de base qui était associée à un $\mathrm{pH}$ ruminal initial bas de 5,41. Des résultats récents (Lettat 2011) chez la vache laitière vont dans le sens de cette hypothèse, puisque la supplémentation en BP seules n'a induit une augmentation du $\mathrm{pH}$ moyen $(+0,24$ unités) qu'avec une ration de base riche en amidon (ensilage de maïs + céréales) induisant un $\mathrm{pH}$ bas $(5,73)$; en revanche, avec une ration de base riche en parois (ensilage d'herbe + pulpe) induisant un pH plus élevé $(5,94)$, la supplémentation en BP n'a pas induit d'augmentation significative du $\mathrm{pH}$ ( $+0,08$ unités).

\section{2 / Effets sur les profils fermen- taires}

Les effets des BP sur les profils fermentaires du rumen sont variables et en moyenne assez faibles (tableau 5). Parmi les différentes variables caractérisant le profil fermentaire, la proportion d'acétate a été significativement réduite suite à l'apport de $\mathrm{BP}$ et $\mathrm{BP}+\mathrm{SC}$, tandis que la concentration en lactate tendait à augmenter avec $\mathrm{BP}+\mathrm{SC}$. En intra-expérience, on observe généralement une augmentation des concentrations en $\mathrm{AGV}$ totaux et en ammoniac, au maximum de $+11 \mathrm{mM}$ et $+43 \mathrm{mg} / \mathrm{L}$ respectivement (Chiquette et al 2008) et une diminution de la proportion d'acétate, au maximum de - 4,8 mol/100 mol (Chiquette 2009) essentiellement au profit du propionate, au maximum $+7,7 \mathrm{~mol} / 100 \mathrm{~mol}$ (Beauchemin et al 2003). Une tendance similaire mais non quantifiée a été rapportée dans d'autres

Figure 2. Variation $\mathrm{du} \mathrm{pH}$ ruminal en fonction $\mathrm{du} \mathrm{pH}$ du rumen du lot témoin (non supplémenté) chez des bovins laitiers et à viande supplémentés en bactéries probiotiques seules (๑) ou associées à la levure S. cerevisiae (๑). Les traitements d'une même étude sont reliés entre eux.

Liste des publications incluses dans la méta-analyse: Van Koevering et al 1994, Ghorbani et al 2002, Nocek et al 2002, Beauchemin et al 2003, Nocek et al 2003, Stein et al 2006, Raeth-Knight et al 2007, Chiquette et al 2008, Lehloenya et al 2008a, Chiquette 2009, Hagg et al 2010, Lettat 2011.

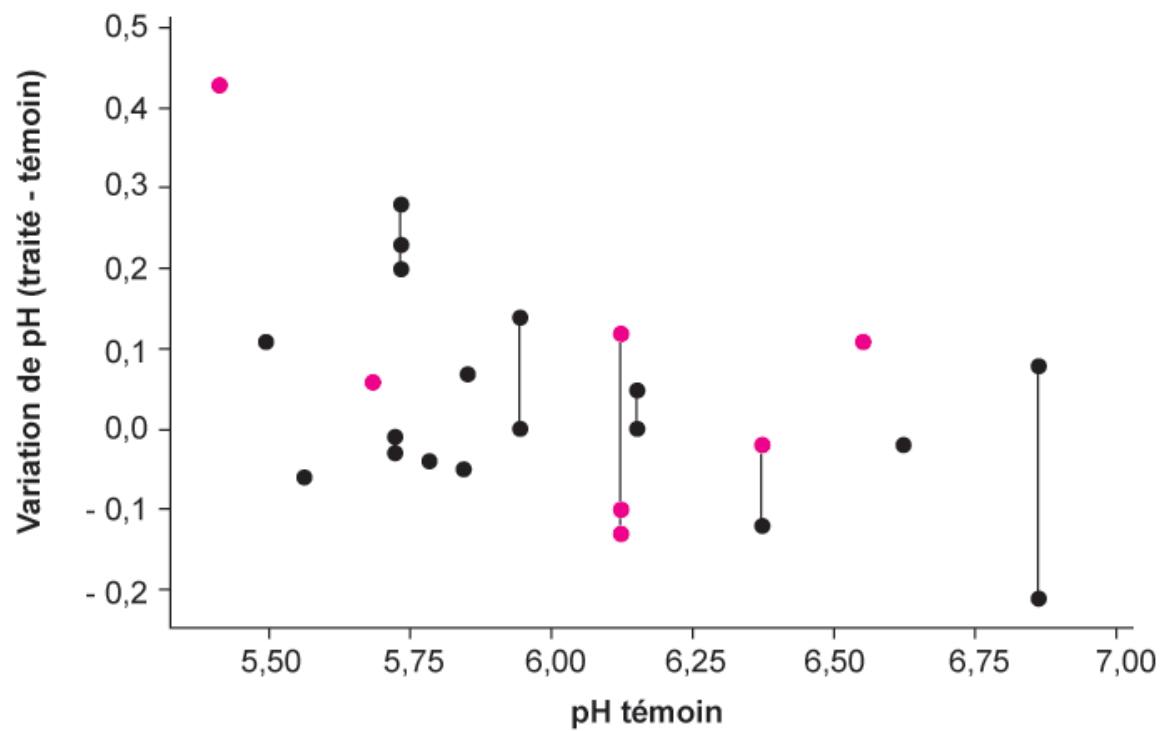


Tableau 5. Effets des bactéries probiotiques seules ou associées à la levure S. cerevisiae sur le pH et les fermentations ruminales.

\begin{tabular}{|c|c|c|c|c|c|c|c|c|}
\hline & \multirow[b]{2}{*}{ Témoin } & \multirow[b]{2}{*}{ Traité } & \multirow[b]{2}{*}{ Variation } & \multirow[b]{2}{*}{$N_{\exp }$} & \multirow[b]{2}{*}{ ETR } & \multicolumn{2}{|c|}{ Effets statistiques } \\
\hline & & & & & & & $\begin{array}{c}\text { Probiotique } \\
\text { (Traité vs } \\
\text { Témoin) }\end{array}$ & $\begin{array}{c}\text { Nature du } \\
\text { probiotique } \\
\text { (Variation BP vs } \\
\text { Variation BP + SC) }\end{array}$ \\
\hline \multirow{2}{*}{$\mathrm{pH}$} & $\mathrm{BP}$ & 6,07 & 6,06 & $-0,02$ & 13 & \multirow{2}{*}{0,1} & \multirow{2}{*}{ NS } & \multirow{2}{*}{$P=0,11$} \\
\hline & $\mathrm{BP}+\mathrm{SC}$ & 6,05 & 6,12 & 0,07 & 7 & & & \\
\hline \multirow{2}{*}{ AGV totaux (mM) } & $\mathrm{BP}$ & 103 & 105 & 1,8 & 9 & \multirow{2}{*}{3,1} & \multirow{2}{*}{ NS } & \multirow{2}{*}{ NS } \\
\hline & $\mathrm{BP}+\mathrm{SC}$ & 110 & 112 & 2,0 & 2 & & & \\
\hline \multirow{2}{*}{ Acétate (\%) } & $\mathrm{BP}$ & 61,1 & 60,4 & $-0,67$ & 11 & \multirow{2}{*}{1,35} & \multirow{2}{*}{$P=0,05$} & \multirow{2}{*}{ NS } \\
\hline & $\mathrm{BP}+\mathrm{SC}$ & 60,4 & 58,3 & $-2,10$ & 3 & & & \\
\hline \multirow{2}{*}{ Propionate (\%) } & $\mathrm{BP}$ & 27,0 & 27,1 & 0,05 & 13 & \multirow{2}{*}{2,46} & \multirow{2}{*}{ NS } & \multirow{2}{*}{ NS } \\
\hline & $B P+S C$ & 22,7 & 24,2 & 1,45 & 2 & & & \\
\hline \multirow{2}{*}{ Butyrate (\%) } & $\mathrm{BP}$ & 11,3 & 11,1 & $-0,17$ & 11 & \multirow{2}{*}{0,86} & \multirow{2}{*}{ NS } & \multirow{2}{*}{ NS } \\
\hline & $\mathrm{BP}+\mathrm{SC}$ & 13,0 & 12,3 & $-0,70$ & 2 & & & \\
\hline \multirow{2}{*}{ Acétate/Propionate } & $\mathrm{BP}$ & 2,63 & 2,51 & $-0,12$ & 11 & \multirow{2}{*}{0,24} & \multirow{2}{*}{ NS } & \multirow{2}{*}{ NS } \\
\hline & $\mathrm{BP}+\mathrm{SC}$ & 2,81 & 2,53 & $-0,28$ & 2 & & & \\
\hline \multirow{2}{*}{ Lactate (mM) } & $\mathrm{BP}$ & 1,87 & 1,59 & $-0,28$ & 7 & \multirow{2}{*}{0,99} & \multirow{2}{*}{ NS } & \multirow{2}{*}{$P=0,09$} \\
\hline & $B P+S C$ & 0,80 & 2,96 & 2,16 & 2 & & & \\
\hline \multirow{2}{*}{$\mathrm{NH}_{3}(\mathrm{mg} / \mathrm{L})$} & $\mathrm{BP}$ & 81,2 & 89,2 & 8,0 & 9 & 06 & NIC & $N C$ \\
\hline & $B P+S C$ & 77,3 & 73,3 & $-3,9$ & 2 & & No & 10 \\
\hline
\end{tabular}

$\mathrm{BP}=$ Bactérie(s) Probiotique(s) seule(s) $; \mathrm{BP}+\mathrm{SC}=$ association BP et levure Saccharomyces cerevisiae ; $\mathrm{N}_{\text {exp }}=$ Nombre d'expériences incluses dans le dispositif ; ETR = Ecart Type Résiduel ; NS = Non Significatif. Les variations significatives par rapport au témoin sont en gras.

Liste des publications incluses dans la méta-analyse : Van Koevering et al 1994, Ghorbani et al 2002, Nocek et al 2002, Beauchemin et al 2003, Nocek et al 2003, Stein et al 2006, Raeth-Knight et al 2007, Chiquette et al 2008, Lehloenya et al 2008a, Chiquette 2009, Hagg et al 2010.

travaux publiés (Weiss et al 2008, Chiquette 2009) ou observée dans un essai récent chez la vache laitière (Lettat 2011). Concernant le lactate, les concentrations ont été soit indétectables (Ghorbani et al 2002, Beauchemin et al 2003, Lehloenya et al 2008a) soit inférieures à $10 \mathrm{mM}$, ce qui correspond à des concentrations compatibles avec l'état d'acidose latente (Martin et al 2006), pour les autres essais. Les rares données disponibles ne permettent pas de conclure sur les effets de l'apport des BP seules ou associées à $S$. cerevisiae.

\section{3 / Effets sur le microbiote ruminal}

Malgré son implication directe dans l'acidose, seulement trois études (Ghorbani et al 2002, Beauchemin et al 2003, Chiquette 2009) ont été recensées sur l'effet des BP sur le microbiote ruminal. Ghorbani et al (2002) ont observé une diminution du nombre de bactéries amylolytiques et une augmentation de la densité des protozoaires suite à l'apport de P. acidopropionici P15, ce qui explique l'augmentation du butyrate dans cette étude (Eugène et al 2004). L'augmentation de la densité des protozoaires est bénéfique car ceux-ci contribuent à stabiliser le $\mathrm{pH}$ et les fermentations ruminales par diverses fonctions (métabolisation du lactate, prédation des bactéries amylolytiques, séquestration de l'amidon...) (Fonty et al 1995, Eugène et al 2004, Morgavi et al 2008). Dans l'étude de Beauchemin et al (2003), aucun effet n'a été observé suite à l'apport de E. faecium $+S$. cerevisiae alors que la supplémentation en E. faecium seule a augmenté le nombre de coliformes et a eu tendance à diminuer le nombre de protozoaires, ce qui constitue un effet négatif pour l'animal. Enfin, dans l'étude de Chiquette (2009), l'effet préventif des BP sur l'acidose n'a pas été associé à une modification des populations cellulolytiques (F. succinogenes,
$R$. albus et $R$. flavefaciens) et/ou utilisatrices de lactate (M. elsdenii). En revanche, nous avons observé récemment (Lettat 2011) que l'amélioration du $\mathrm{pH}$ était associée à une augmentation de la densité bactérienne totale et des proportions de Prevotella et $R$. albus. En résumé, les mécanismes d'action des bactéries probiotiques utilisées dans l'alimentation des ruminants pour prévenir l'acidose ruminale sont encore difficiles à comprendre.

\section{Conclusion}

Cette analyse quantitative de la littérature nous a permis de préciser l'intérêt des bactéries probiotiques en tant qu'additif alimentaire pour moduler les fermentations microbiennes du rumen et améliorer les performances des bovins en production. Les résultats montrent que la supplémentation des 
rations en $\mathrm{BP}$, utilisées seules ou en association avec les levures, peut améliorer de façon modérée les performances animales (lait, viande). Il est important de noter que la présence de tampons et/ou de promoteurs de croissance dans les rations peut également avoir contribué à cet effet.

Il est difficile d'expliquer la variabilité de l'efficacité des bactéries probiotiques pour stabiliser le $\mathrm{pH}$ ruminal compte tenu du faible nombre d'études, de la variabilité des conditions expérimentales (animaux, types bactériens, doses) et du fait que l'essentiel des rations étudiées étaient supplémentées avec des tampons. Les effets bénéfiques les plus marqués ont été observés chez la vache laitière avec des associations de bactéries lactiques et de levures, lorsque le $\mathrm{pH}$ moyen induit par la ration de base était bas. Il est donc possible que ces effets ne soient pas spécifiques des bactéries probiotiques, mais des levures, dont les effets ont déjà été rapportés dans la littérature. Une action synergique des deux types microbiens n'est pas à exclure.

Nos données expérimentales récentes obtenues sur vaches laitières confirment que l'efficacité des bactéries probio- tiques dépend du $\mathrm{pH}$ ruminal initial et sont les premières à démontrer l'efficacité spécifique des bactéries lactiques et/ou propioniques pour limiter les risques d'acidose ruminale latente. L'ensemble des résultats présentés dans cet article sont prometteurs et devront être confirmés par des recherches complémentaires sur le long terme et impliquant un plus grand nombre d'animaux laitiers ou à viande, afin de confirmer les tendances observées par cette métaanalyse et préciser les mécanismes d'action des bactéries probiotiques. Par ailleurs, il serait pertinent de tester l'effet dose et la synergie bactéries-levures.

\section{Références}

Beauchemin K.A., Yang W.Z., Morgavi D.P., Ghorbani G.R., Kautz W., Leedle J.A., 2003. Effects of bacterial direct-fed microbials and yeast on site and extent of digestion, blood chemistry, and subclinical ruminal acidosis in feedlot cattle. J. Anim. Sci., 81, 1628-1640.

Block E., Nocek J.E., Kautz W.P., Leedle J.A.Z., 2000. Direct fed microbial and anionic salt supplementation to dairy cows fed 21 days pre- to 70 days postpartum. J. Anim. Sci. 78, Suppl. 1, 304.

Brashears M.M., Galyean M.L., Loneragan G.H., Mann J.E., Killinger-Mann K., 2003. Prevalence of Escherichia coli O157:H7 and performance by beef feedlot cattle given Lactobacillus direct-fed microbials. J. Food Prot., 66, 748-754.

Brockman R.P., 1990. Effect of insulin on the utilization of propionate in gluconeogenesis in sheep. Br. J. Nutr., 64, 95-101.

Chaucheyras-Durand F., Walker N.D., Bach A., 2008. Effects of active dry yeasts on the rumen microbial ecosystem: Past, present and future. Anim. Feed Sci. Technol., 145, 5-26.

Chaucheyras-Durand F., Durand H., 2010. Probiotics in animal nutrition and health. Beneficial Microbes., 1, 3-9.

Chiquette J., 2009. Evaluation of the protective effect of probiotics fed to dairy cows during a subacute ruminal acidosis challenge. Anim. Feed Sci. Technol., 153, 278-291.

Chiquette J., Allison M.J., Rasmussen M.A., 2008. Prevotella bryantii 25A used as a probiotic in early-lactation dairy cows: effect on ruminal fermentation characteristics, milk production, and milk composition. J. Dairy Sci., 91, 35363543.

de Ondarza M.B., Seymour W.M., 2008. Case study: Effect of propionibacteria supplementation on yield of milk and milk components of dairy cows. Prof. Anim. Scientist., 24, 254-259.

Desnoyers M., Giger-Reverdin S., Bertin G., Duvaux-Ponter C., Sauvant D., 2009. Metaanalysis of the influence of Saccharomyces cerevisiae supplementation on ruminal parameters and milk production of ruminants. J. Dairy Sci., 92, 1620-1632.

Elam N.A., Gleghorn J.F., Rivera J.D., Galyean M.L., Defoor P.J., Brashears M.M.,
2003. Effects of live cultures of Lactobacillus acidophilus (strains NP45 and NP51) and Propionibacterium freudenreichii on performance, carcass, and intestinal characteristics, and Escherichia coli strain O157 shedding of finishing beef steers. J. Anim. Sci., 81, 2686-2698.

Eugène M., Archimède H., Sauvant D., 2004. Quantitative meta-analysis on the effects of defaunation of the rumen on growth, intake and digestion in ruminants. Livest. Prod. Sci., 85, 81-97.

Fonty G., Jouany J.P., Forano E., Gouet P., 1995. L'écosysteme microbien du réticulorumen. In: Jarrige R., Ruckebusch Y., Demarquilly C., Farce M.H., Journet M. (Eds) : Nutrition des ruminants domestiques, ingestion et digestion. INRA, Paris, France, 299-347.

Francisco C.C., Chamberlain C.S., Waldner D.N., Wettemann R.P., Spicer L.J., 2002. Propionibacteria fed to dairy cows: effects on energy balance, plasma metabolites and hormones, and reproduction. J. Dairy Sci., 85, 1738-1751.

Galyean M.L., Nunnery G.A., Defoor P.J., Salyer G.B., Parsons C.H., 2000. Effects of live cultures of Lactobacillus acidophilus (Strains 45 and 51) and Propionibacterium freudenreichii PF-24 on performance and carcass characteristics of finishing beef steers. Burnett Center Progress Report No 8. 12p. http://www.afs.ttu.edu/burnett center/progress reports/bc8.pdf

Ghorbani G.R., Morgavi D.P., Beauchemin K.A., Leedle J.A., 2002. Effects of bacterial direct-fed microbials on ruminal fermentation, blood variables, and the microbial populations of feedlot cattle. J. Anim. Sci., 80, 1977-1985.

Gomez-Basauri J., de Ondarza M.B. Siciliano-Jones J., 2001. Intake and milk production of dairy cows fed lactic acid bacteria and mannanoligosaccharide. J. Dairy Sci., 84, Suppl. 1, 283.

Gozho G.N., Plaizier J.C., Krause D.O., Kennedy A.D., Wittenberg K.M., 2005. Subacute ruminal acidosis induces ruminal lipopolysaccharide endotoxin release and triggers an inflammatory response. J. Dairy Sci., 88, 1399-1403.

Gozho G.N., Krause D.O., Plaizier J.C., 2006. Rumen lipopolysaccharide and inflam- mation during grain adaptation and subacute ruminal acidosis in steers. J. Dairy Sci., 89, 4404-4413.

Gozho G.N., Krause D.O., Plaizier J.C., 2007. Ruminal lipopolysaccharide concentration and inflammatory response during graininduced subacute ruminal acidosis in dairy cows. J. Dairy Sci., 90, 856-866.

Hagg F.M., Erasmus L.J., Henning P.H., Coertze R.J., 2010. The effect of a direct fed microbial (Megasphera elsdenii) on the productivity and health of Holstein cows. S. Afr. J. Anim. Sci., 40, 101-112.

Jaquette R.D., Dennis R.J., Coalson J.A., Ware D.R., Manfredi E.T., Read P.L., 1988. Effect of feeding viable Lactobacillus acidophilus (BT1386) on performance of lactating dairy cows. J. Dairy Sci., 71, Suppl. 1, 219.

Khafipour E., Krause D.O., Plaizier J.C., 2009a. Alfalfa pellet-induced subacute ruminal acidosis in dairy cows increases bacterial endotoxin in the rumen without causing inflammation. J. Dairy Sci., 92, 1712-1724.

Khafipour E., Krause D.O., Plaizier J.C., 2009b. A grain-based subacute ruminal acidosis challenge causes translocation of lipopolysaccharide and triggers inflammation. J. Dairy Sci., 92, 1060-1070.

Kim S.W., Standford D.G., Roman-Rosario H., Yokoyama M.T., Rust S. R., 2000. Potential use of Propionibacterium acidipropionici, strain DH42, as a direct-fed microbial for cattle. J. Anim. Sci., 78, Suppl. 1, 1225.

Kleen J.L., Hooijer G.A., Rehage J., Noordhuizen J.P.T.M., 2003. Subacute ruminal acidosis (SARA): A review. J. Vet. Med. A., $50,406-414$.

Kleen J.L., Hooijer G.A., Rehage J., Noordhuizen J.P.T.M., 2009. Subacute ruminal acidosis in Dutch dairy herds. Vet. Rec., 164, 681-684.

Komari R.K., Reddy Y.K.L., Suresh J., Raj D.N., 1999. Effect of feeding yeast culture (Saccharomyces cerevisae) and Lactobacillus acidophilus on production performance of crossbred dairy cows. J. Dairy Sci., 82, Suppl. 1,128 .

Krause K.M., Dhuyvetter D.V., Oetzel G.R., 2009. Effect of a low-moisture buffer block on ruminal $\mathrm{pH}$ in lactating dairy cattle induced 
with subacute ruminal acidosis. J. Dairy Sci., $92,352-364$

Lehloenya K.V., Krehbiel C.R., Mertz K.J., Rehberger T.G., Spicer L.J., 2008a. Effects of propionibacteria and yeast culture fed to steers on nutrient intake and site and extent of digestion. J. Dairy Sci., 91, 653-662.

Lehloenya K.V., Stein D.R., Allen D.T., Selk G.E., Jones D.A., Aleman M.M., Rehberger T.G., Mertz K.J., Spicer L.J., 2008b. Effects of feeding yeast and propionibacteria to dairy cows on milk yield and components, and reproduction. J. Anim. Physiol. Anim. Nutr., 92, 190-202.

Lettat A., 2011. Efficacité et mode d'action des bactéries propioniques et/ou lactiques pour prévenir l'acidose latente chez le ruminant. Thèse, Ecole Doctorale SVSAE, Université Blaise Pascal, Clermont-Ferrand, 209p.

Loncke C., Ortigues-Marty I., Vernet J., Lapierre H., Sauvant D., Noziere P., 2009. Empirical prediction of net portal appearance of volatile fatty acids, glucose, and their secondary metabolites ( $\beta$-hydroxybutyrate, lactate) from dietary characteristics in ruminants: A meta-analysis approach. J. Anim Sci., 87, 253268.

Martin C., Brossard L., Doreau M., 2006. Mécanismes d'apparition de l'acidose ruminale latente et conséquences physiopathologiques et zootechniques. INRA Prod. Anim., 19, 93108 .

Meissner H.H., Henning P.H., Horn C.H., Leeuw K.J., Hagg F.M., Fouché G., 2010. Ruminal acidosis: a review with detailed reference to the controlling agent Megasphaera elsdenii NCIMB 41125. S. Afr. J. Anim. Sci., 40, 79-100.

Meschy F., Bravo D., Sauvant D., 2004. Analyse quantitative des réponses des vaches laitières à l'apport de substances tampon. INRA Prod. Anim., 17, 11-18.

Morgavi D.P., Jouany J.P., Martin C., 2008. Changes in methane emission and rumen fermentation parameters induced by refaunation in sheep. Aust. J. Exp. Agric., 48, 69-72.
Nocek J.E., 1997. Bovine acidosis: Implications on laminitis. J. Dairy Sci., 80, 1005-1028.

Nocek J.E., Kautz W.P., 2006. Direct-fed microbial supplementation on ruminal digestion, health, and performance of pre- and postpartum dairy cattle. J. Dairy Sci., 89, 260-266.

Nocek J.E., Kautz W.P., Leedle J.A.Z., Allman J.G., 2002. Ruminal supplementation of direct-fed microbials on diurnal $\mathrm{pH}$ variation and in situ digestion in dairy cattle. J. Dairy Sci., 85, 429-433.

Nocek J.E., Kautz W.P., Leedle J.A.Z., Block E., 2003. Direct-fed microbial supplementation on the performance of dairy cattle during the transition period. J. Dairy Sci., 86, 331-335.

Peterson R.E., Klopfenstein T.J., Erickson G.E., Folmer J., Hinkley S., Moxley R.A., Smith D.R., 2007. Effect of Lactobacillus acidophilus strain NP51 on Escherichia coli O157:H7 fecal shedding and finishing performance in beef feedlot cattle. J. Food Prot., 70, 287-291.

Peyraud J.L., Apper-Bossard E., 2006. L'acidose latente chez la vache laitière. INRA Prod. Anim., 19, 79-92.

Raeth-Knight M.L., Linn J.G., Jung H.G., 2007. Effect of direct-fed microbials on performance, diet digestibility, and rumen characteristics of Holstein dairy cows. J. Dairy Sci. 90, 1802-1809.

Rust S.R., Metz K., Ware D.R., 2000. Effects of Bovamine ${ }^{\mathrm{TM}}$ rumen culture on the performance and carcass characteristics of feedlot steers. J. Anim. Sci., 78, Suppl. 2, 83.

Sauvant D., Peyraud J.L., 2010. Calculs de ration et évaluation du risque acidogène. INRA Prod. Anim., 23, 333-342.

Sauvant D., Giger-Reverdin S., Meschy F., 2006. Le contrôle de l'acidose ruminale latente. INRA Prod. Anim., 19, 69-78.

Stein D.R., Allen D.T., Perry E.B., Bruner J.C., Gates K.W., Rehberger T.G., Mertz K., Jones D., Spicer L.J., 2006. Effects of feeding propionibacteria to dairy cows on milk yield, milk components, and reproduction. J. Dairy Sci., 89, 111-125.

Stephens T.P., Loneragan G.H., Chichester L.M., Brashears M.M., 2007a. Prevalence and enumeration of Escherichia coli $\mathrm{O} 157$ in steers receiving various strains of Lactobacillusbased direct-fed microbials. J. Food Prot., 70, 1252-1255.

Stephens T.P., Loneragan G.H., Karunasena E., Brashears M.M., 2007b. Reduction of Escherichia coli $\mathrm{O} 157$ and Salmonella in feces and on hides of feedlot cattle using various doses of a direct-fed microbial. J. Food Prot., 70, 2386-2391.

Swinney-Floyd D., Gardner B.A., Owens F.N., Rehberger T.G., Parrott T.D., 1999 Effects of inoculation with either Propionibacterium strain P-63 alone or combined with Lactobacillus acidophilus strain LA53545 on performance of feedlot cattle. J. Anim. Sci., 77, Suppl. 1, 77-78.

Van Koevering, M.T., Owens F.N., Secrist D.S., Anderson R.H., Herman R.E., 1994. Cobactin II for feedlot steers. J. Anim. Sci., 72, Suppl. 1, 83 .

Vasconcelos J.T., Elam N.A., Brashears M.M., Galyean M.L., 2008. Effects of increasing dose of live cultures of Lactobacillus acidophilus (Strain NP 51) combined with a single dose of Propionibacterium freudenreichii (Strain NP 24) on performance and carcass characteristics of finishing beef steers. J. Anim. Sci., 86, 756-762.

Ware D.R., Read P.L., Manfredi E.T., 1988. Lactation performance of two large dairy herds fed Lactobacillus acidophilus strain BT138 in a switchback experiment. J. Dairy. Sci., 71, Suppl. 1, 219.

Weiss W.P., Wyatt D.J., McKelvey T.R., 2008. Effect of feeding propionibacteria on milk production by early lactation dairy cows. J. Dairy Sci., 91, 646-652.

West J.W., Bernard J.K., Cross G.H., Trammell D.S., 2005. Effects of Live Bacterial Inoculants on Performance of Lactating Dairy Cows. http://www.bovamine.com/publica$\underline{\text { tions/Georgia Final report.pdf }}$

\section{Résumé}

L'acidose ruminale latente est une préoccupation majeure chez les ruminants à potentiel élevé de production. Sa prévention par la supplémentation des rations avec des Bactéries Probiotiques (BP) est une stratégie potentiellement intéressante compte tenu de leur capacité à s'adapter à l'environnement très compétitif du rumen. Une méta-analyse des données publiées regroupant 33 expériences (82 traitements) a permis de mettre en évidence l'effet des BP, seules ou associées à la levure Saccharomyces cerevisiae (BP + SC), sur les fermentations dans le rumen et les performances zootechniques de bovins laitiers et à viande. Chez ces derniers la supplémentation en BP ou en BP + SC n'a pas affecté en moyenne les performances animales. En revanche, chez la vache laitière la supplémentation en BP + SC a permis d'augmenter l'ingestion $(+1,7 \mathrm{~kg} / \mathrm{j})$ et la production de lait $(+2,36 \mathrm{~kg} / \mathrm{j})$ sans modifier sa composition. L'effet des probiotiques sur les fermentations dans le rumen est très variable et en moyenne relativement faible. La régulation du pH ruminal moyen par les BP seules ou les BP + SC n'est effective que lorsque la ration de base entraîne un pH ruminal inférieur à 5,75. Enfin, bien que non pris en compte dans le dispositif statistique du fait d'un nombre limité de données, les réponses des performances animales semblent également dépendre du type de BP employées. 


\begin{abstract}
Quantitative analysis of the effect of bacterial probiotics on rumen fermentations and performances in dairy and beef cattle

Subacute ruminal acidosis is a major concern in nutrition of high-producing ruminants. Its prevention by supplementing diets with probiotic bacteria (PB) is a potentially effective strategy that should be considered in light of the ability of probiotics to positively modulate the ruminal flora and fermentations. A meta-analysis of 33 experiments (82 treatments) was made to highlight the effect of $\mathrm{PB}$, used alone or associated with the yeast Saccharomyces cerevisiae $(\mathrm{PB}+\mathrm{SC})$, on ruminal parameters and animal performance of dairy and beef cattle. Although in beef cattle supplementation with PB or with PB + SC did not affect the average animal performance, in dairy cows, supplementation with $\mathrm{PB}+\mathrm{SC}$ increased intake $(+1.7 \mathrm{~kg} /$ day $)$ and milk production $(+2.36 \mathrm{~kg} / \mathrm{day})$ without affecting milk composition. The effect of probiotics on ruminal fermentations was inconsistent and relatively weak. However, for low initial mean $\mathrm{pH}$ values $(\mathrm{pH}<5.75)$, supplementation with $\mathrm{PB}$ alone or $\mathrm{PB}+\mathrm{SC}$ significantly increased the pH. Finally, although not included in the statistical model because of limited data, animal responses also appear to depend on the type of PB used.
\end{abstract}

LETTAT A., MARTIN C., BERGER C., NOZIÈRE P., 2012. Analyse quantitative de l'effet des bactéries probiotiques sur les fermentations dans le rumen et les performances des bovins en production. INRA Prod. Anim., 25, 4, 351-360. 